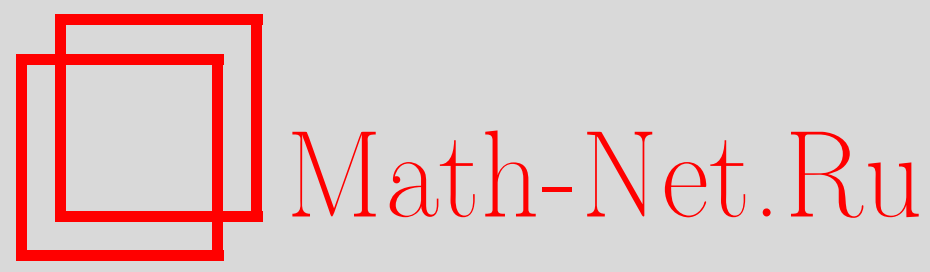

Н. Г. Мощевитин, И. Д. Шкредов, О критерии нормальности Пятецкого-Шапиро, Матем. заметки, 2003, том 73, выпуск 4, 577-589

DOI: https://doi.org/10.4213/mzm206

Использование Общероссийского математического портала Math-Net.Ru подразумевает, что вы прочитали и согласны с пользовательским соглашением http://www . mathnet.ru/rus/agreement

Параметры загрузки:

IP : 54.80 .97 .219

26 апреля 2023 г., $17: 51: 07$

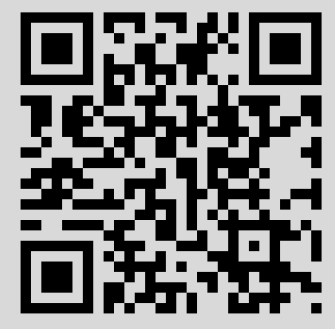




\title{
О КРИТЕРИИ НОРМАЛЬНОСТИ ПЯТЕЦКОГО-ШАПИРО
}

\author{
Н. Г. Мощевитин, И. Д. Шкредов
}

В данной работе критерию нормальности Постникова-Пятецкого придана более современная форма и получены его неулучшаемые аналоги для конечных цепей Маркова, цепных дробей и обобщенного сдвига Бернулли.

Библиографоия: 13 названий.

1. Введение. Пусть $X$ - пространство с сигма-алгеброй измеримых множеств $\Phi$ и мерой $\mu$, а $T$ - измеримое эргодическое отображение пространства $X$ в себя, сохраняющее эту меру. Всюду ниже будем считать, что $\mu(X)=1$.

Для произвольной измеримой функции $f(x)$ рассмотрим биркгофовское среднее

$$
S_{l}(T, x, f)=S_{l}(x, f)=\sum_{m=0}^{l-1} f\left(T^{m} x\right) .
$$

Пусть $\left\{C_{m}\right\}$ - не более чем счетное семейство измеримых подмножеств $X$. Определим аналог хаусдорфовой меры $H(\cdot)$ для множества $E$ относительно этого семейства, как $\inf \left\{\sum \mu\left(C_{i}\right)\right\}$, где inf берется по не более чем счетным покрытиям $E$. Будем говорить, что меры $\mu$ и $H$ согласованы, если любое $\mu$-измеримое множество является $H$-измеримым.

Обозначим через $\Gamma$ семейство $\mu$-измеримых множеств $\{V\}$, которые с любой точностью аппроксимируются множествами семейства $\left\{C_{m}\right\}$. (То есть для произвольного $V$ из $Г$ и любого $\varepsilon>0$ существуют наборы непересекающихся множеств $\left\{M_{i}\right\}$ и $\left\{N_{i}\right\}$ из семейства $\left\{C_{m}\right\}$, так что $\bigsqcup M_{i} \subseteq V \subseteq \bigsqcup N_{i}$ и $\left.\sum \mu\left(N_{i}\right)-\varepsilon<\mu(V)<\sum \mu\left(M_{i}\right)+\varepsilon\right)$.

Пусть $\chi_{I}-$ характеристическая функция измеримого множества $I$. Знаменитая эргодическая теорема Биркгофа (см., например, [1]) утверждает, что для почти всех точек $x_{0}$ вьполнено

$$
\lim _{\nu \rightarrow \infty} \frac{S_{\nu}\left(x_{0}, \chi_{I}\right)}{\nu}=\mu(I) .
$$

В работе [2] Пятецким-Шапиро для динамической системы, порожденной отображением $x \mapsto\{q x\}, q \in \mathbb{N}, q>1$, интервала $[0,1)$ в себя, был дан критерий того, чтобы для данной точки $x_{0}$ вьполнялось $(1)$. Ряд последующих работ Пятецкого-Шапиро и Постникова [2]-[5] и других (библиографию см. в [4], [5]) содержал обобщения и усиления первоначального критерия из работы [2].

Достаточно общая формулировка выглядит следующим образом (ср. с [5, теорема 6]).

Работа выполнена при поддержке Российского фонда фундаментальных исследований, грант № 02-01-00912, и фонда INTAS. 
Теорема А. Пусть точка $x_{0} \in X$. Пусть меры $\mu$ и $Н$ согласованы $и$ для любого $\mu$-измеримого $А$ выполнено $\mu(A)=H(A)$. Если существует полохительная постоянная $C$, что для любого мнохества $I$ из семейства $\left\{C_{m}\right\}$ выполняется

$$
\limsup _{\nu \rightarrow \infty} \frac{S_{\nu}\left(x_{0}, \chi_{I}\right)}{\nu} \leqslant C \mu(I)
$$

то для любого множества I из Г выполняется

$$
\lim _{\nu \rightarrow \infty} \frac{S_{\nu}\left(x_{0}, \chi_{I}\right)}{\nu}=\mu(I) .
$$

Пусть $q$-целое число, $q>1, \alpha$ - вешественное число. Обозначим через $P_{\nu}(\alpha, \Delta)$, где $\Delta-$ некоторьй интервал из отрезка $[0,1]$, число попаданий в $\Delta$ дробных долей $\left\{\alpha q^{k}\right\}$, $k=0,1, \ldots, \nu-1$.

Для динамической системы, соответствующей распределению дробных долей $\left\{\alpha q^{k}\right\}$, с $X=[0,1)$ и $T x=\{q x\}$ в работе [3] Пятецким-Шапиро был доказан результат, значительно улучшающий общую теорему А.

ТЕОРемА Б. Пусть $\varphi(t)$ - монотонно возрастающая полоэсительнозначная функиия такая, что для любого $\varepsilon>0$ выполняется

$$
\liminf _{t \rightarrow 0} \frac{\varphi(t)}{t^{1-\varepsilon}}=0
$$

Если для любого интервала $\Delta$ выполняется

$$
\limsup _{\nu \rightarrow \infty} \frac{P_{\nu}(\alpha, \Delta)}{\nu} \leqslant \varphi(|\Delta|)
$$

то дробные доли $\left\{\alpha q^{k}\right\}$ равномерно распределены.

Как известно, хаусдорфова размерность множества чисел, не являющихся нормальными по основанию $q$, равна 1. (Более того, как показал Шмидт [6], хаусдорфова размерность множества чисел, не являющихся нормальными ни по какому основанию, равна единице.) С другой стороны, теорема Б означает, что хаусдорфова мера чисел, не нормальных по фиксированному основанию $q$, относительно функции $\varphi$ равна 0 .

В данной заметке теорема Б перенесена на некоторые другие динамические системы (в том числе и рассматривавшиеся в [4]), при этом функция $\varphi$ будет выбираться так, чтобы хаусдорфова мера “исключительного” (в смысле эргодической теоремы) множества точек была равна 0 .

2. Общая постановка задачи. Возьмем произвольное $\delta>0$ и произвольное натуральное $l$. Рассмотрим множества

$$
A_{l}(T, f, \delta)=A_{l}(f, \delta)=\left\{x \in X:\left|\frac{S_{l}(T, x, f)}{l}-\int f d \mu\right|>\delta\right\}
$$

Как утверждает статистическая эргодическая теорема, для любого $\delta>0$ мера множеств $A_{l}(f, \delta)$ стремится к нулю при $l \rightarrow \infty$. (По поводу скорости сходимости см. [7], [8]). 
Пусть $\left\{C_{m}\right\}$ - не более чем счетное семейство измеримьх подмножеств $X$, а $\varphi(t)-$ монотонно возрастающая положительнозначная функция аргуметна $t \in \mathbb{R}_{+}$. Определим меру $H_{\varphi}(\cdot)$ для множества $E$ относительно этого семейства, как $\inf \left\{\sum \varphi\left(\mu\left(C_{i}\right)\right)\right\}$, где inf берется по не более чем счетным покрытиям $E$. (При $\varphi(t)=t$ мера $H_{\varphi}$ совпадает с рассматривавшейся вьше мерой $H$.)

Мы сформулируем критерий Пятецкого для произвольной эргодической динамической системы и проведем доказательство, аналогичное доказательству из [2].

Теорема 1. Пусть точка $x_{0} \in X$. Если для произвольного множества I из семейства $\left\{C_{m}\right\}$ выполнено

$$
\limsup _{\nu \rightarrow \infty} \frac{S_{\nu}\left(x_{0}, \chi_{I}\right)}{\nu} \leqslant \varphi(\mu(I))
$$

и для любого $\delta>0$ выполнено $H_{\varphi}\left(A_{l}\left(T, \chi_{I}, \delta\right)\right) \rightarrow 0$ при $l \rightarrow \infty$, то для произвольного множсества $V$ из Г имет место асимптотическое равенство

$$
\lim _{\nu \rightarrow \infty} \frac{S_{\nu}\left(x_{0}, \chi_{V}\right)}{\nu}=\mu(V)
$$

ДокАЗАТЕЛЬСТВо. Возьмем любое $\delta>0$ и характеристическую функцию $\chi$ произвольного множества $I$ из семейства $\left\{C_{m}\right\}$. Для любых натуральных $\nu$ и $l$ справедлива формула

$$
S_{\nu}\left(x_{0}, \chi\right)=\frac{1}{l} \sum_{t=0}^{\nu-1} S_{l}\left(T^{t} x_{0}, \chi\right)+O(l) .
$$

Из этой формулы получаем

$$
\begin{aligned}
\left|\frac{S_{\nu}\left(x_{0}, \chi\right)}{\nu}-\mu(I)\right| & =\frac{1}{\nu}\left|\sum_{t=0}^{\nu-1}\left(\frac{S_{l}\left(T^{t} x_{0}, \chi\right)}{l}-\mu(I)\right)\right|+O\left(\frac{l}{\nu}\right) \\
& \leqslant \frac{1}{\nu} \sum_{t}^{1}\left|\frac{S_{l}\left(T^{t} x_{0}, \chi\right)}{l}-\mu(I)\right|+\frac{1}{\nu} \sum_{t}^{2}\left|\frac{S_{l}\left(T^{t} x_{0}, \chi\right)}{l}-\mu(I)\right|+O\left(\frac{l}{\nu}\right) \\
& \leqslant \delta+\frac{2 R_{\nu}}{\nu}+O\left(\frac{l}{\nu}\right)
\end{aligned}
$$

где суммирование в первой сумме распространено на те слагаемые, для которых

$$
\left|\frac{S_{\nu}\left(T^{t} x_{0}, \chi\right)}{l}-\mu(I)\right| \leqslant \delta,
$$

a $R_{\nu}$ обозначает количество слагаемых во второй сумме, для которых

$$
\left|\frac{S_{\nu}\left(T^{t} x_{0}, \chi\right)}{l}-\mu(I)\right|>\delta .
$$


Итак, для индексов $t$ из области суммирования в $\sum^{2}$ выполняется $T^{t} x_{0} \in A_{l}(\chi, \delta)$. Возьмем покрытие $A_{l}(\chi, \delta)$ множествами из семейства $\left\{C_{m}\right\}$, так что

$$
\sum_{i} \varphi\left(\mu\left(C_{i}\right)\right)<H_{\varphi}\left(A_{l}(\chi, \delta)\right)+\delta
$$

По условию теоремы $H_{\varphi}\left(A_{l}(\chi, \delta)\right) \rightarrow 0$ при $l \rightarrow \infty$, значит

$$
\limsup _{\nu \rightarrow \infty} \frac{R_{\nu}}{\nu} \leqslant \sum_{i} \varphi\left(\mu\left(C_{i}\right)\right)<2 \delta
$$

при достаточно больших $l$. Значит,

$$
\limsup _{\nu \rightarrow \infty}\left|\frac{S_{\nu}\left(x_{0}, \chi\right)}{\nu}-\mu(I)\right|=0
$$

т.e.

$$
\lim _{\nu \rightarrow \infty}\left|\frac{S_{\nu}\left(x_{0}, \chi\right)}{\nu}-\mu(I)\right|=0 .
$$

Пусть теперь $\chi$-характеристическая функция произвольного множества $V$ из Г. Для всякого положительного $\varepsilon$ найдется набор множеств $N_{i}$ из семейства $\left\{C_{m}\right\}$ таких, что $V \subseteq \bigsqcup N_{i}$ и $\mu(V)>\sum \mu\left(N_{i}\right)-\varepsilon$. Теперь имеем

$$
\limsup _{\nu \rightarrow \infty} \frac{S_{\nu}\left(T, x_{0}, \chi\right)}{\nu} \leqslant \sum \limsup _{\nu \rightarrow \infty} \frac{S_{\nu}\left(T, x_{0}, \chi_{i}\right)}{\nu}=\sum \mu\left(N_{i}\right)<\mu(V)+\varepsilon
$$

где $\chi_{i}=\chi_{N_{i}}$. Итак,

$$
\limsup _{\nu \rightarrow \infty} \frac{S_{\nu}\left(T, x_{0}, \chi\right)}{\nu} \leqslant \mu(V)
$$

Аналогично,

$$
\liminf _{\nu \rightarrow \infty} \frac{S_{\nu}\left(T, x_{0}, \chi\right)}{\nu} \geqslant \mu(V) .
$$

Значит, для $\chi$ вьполнено (3). Теорема доказана.

Часто оказьвается, что семейство $\left\{C_{m}\right\}$ имеет специальный вид.

Пусть $\xi$ - не более чем счетное измеримое разбиение пространства $X$. Если $\xi$ и $\eta-$ два таких разбиения, то их совместное разбиение определяется следующим образом: $\xi \vee \eta:=\{A \cap B: A \in \xi, B \in \eta\}$. Для измеримого разбиения $\xi$, сохраняющего меру преобразования $T$, и произвольного натурального $n$ определим итерированное разбиение $\xi_{-n}^{T}:=\xi \vee T^{-1} \xi \vee \cdots \vee T^{-n+1} \xi, \xi_{0}^{T}=\xi$.

Мы будем считать, что семейство $\left\{C_{m}\right\}$ есть совокупность всех элементов разбиений $\xi_{-\infty}^{T}$. Множество всех этих элементов иногда обозначают через $\xi_{-\infty}^{T}=\left\{\xi_{-n}^{T}\right\}_{n=0}^{\infty}$ (эта ситуация была фактически рассмотрена в [4]). Как и прежде, Г обозначает семейство $\mu$-измеримых множеств $\{V\}$, которые с любой точностью аппроксимируются множествами семейства $\left\{C_{m}\right\}$. 
Tеорема 1'. Пусть точка $x_{0} \in X$. Если для любого $n$ и характеристической функиии $\chi_{I}$ произвольного әлемента $I \in \xi_{-n}^{T}$ выполняется

$$
\limsup _{\nu \rightarrow \infty} \frac{S_{\nu}\left(x_{0}, \chi_{I}\right)}{\nu} \leqslant \varphi(\mu(I))
$$

$u$ кроме того, для любого $\delta>0$ выполняется $H_{\varphi}\left(A_{l}\left(T^{n}, \chi_{I}, \delta\right)\right) \rightarrow 0$ при $l \rightarrow \infty$, то для характеристической функчии ұ произвольного множества $V \in \Gamma$

$$
\lim _{\nu \rightarrow \infty} \frac{S_{\nu}\left(x_{0}, \chi\right)}{\nu}=\mu(V) .
$$

ДокАЗАТЕЛЬСТво. По теореме 1 для любого элемента начального разбиения $\xi$ выполнено (8). Пусть $\chi$ - характеристическая функция множества $K=E_{1} \cap T^{-1} E_{2} \cap \cdots$ $\cap T^{-k+1} E_{k}, E_{i} \in \xi$, которое является элементом разбиения $\xi_{-k}^{T}$ для некоторого $k$. Рассмотрим преобразование $T^{k}$ и будем считать разбиение $\eta=\xi_{-k}^{T}$ начальным для этой новой динамической системы. Возьмем точки $x_{0}, T x_{0}, \ldots, T^{k-1} x_{0}$. Для них вьполнено условие (7) теоремы. Очевидно, что для преобразования $T^{k}$ и этих точек вьполнено

$$
\limsup _{\nu \rightarrow \infty} \frac{S_{\nu}\left(T^{k}, T^{j} x_{0}, \chi_{I}\right)}{\nu} \leqslant k \cdot \varphi(\mu(I)), \quad j=0, \ldots, k-1 .
$$

Меры $H_{\varphi}$ и $H_{k \varphi}$ стремятся к нулю одновременно. Таким образом, все условия теоремы 1 для динамической системы, порожденной преобразованием $T^{k}$, с начальным разбиением $\eta$ выполнены. Применяя формулу

$$
S_{\nu}(T, x, \chi)=\sum_{j=0}^{k-1} S_{[\nu / k]}\left(T^{k}, T^{j} x, \chi\right)+O(k),
$$

получаем

$$
\lim _{\nu \rightarrow \infty} \frac{S_{\nu}\left(T, x_{0}, \chi\right)}{\nu}=\sum_{j=0}^{k-1} \frac{1}{k} \lim _{\nu \rightarrow \infty} \frac{S_{[\nu / k]}\left(T^{k}, T^{j} x_{0}, \chi\right)}{[\nu / k]}=\sum_{j=0}^{k-1} \frac{1}{k} \mu(K)=\mu(K) .
$$

Далее будем действовать также, как и в конце доказательства теоремы 1. Пусть теперь $\chi$ - характеристическая функция произвольного множества $V$ из $\Gamma$. Для всякого положительного $\varepsilon$ найдется набор множеств $N_{i}$ из семейства $\left\{C_{m}\right\}$ таких, что $V \subseteq \bigsqcup N_{i}$ и $\mu(V)>\sum \mu\left(N_{i}\right)-\varepsilon$. Теперь снова

$$
\limsup _{\nu \rightarrow \infty} \frac{S_{\nu}\left(T, x_{0}, \chi\right)}{\nu} \leqslant \mu(V)+\varepsilon .
$$

Аналогично,

$$
\liminf _{\nu \rightarrow \infty} \frac{S_{\nu}\left(T, x_{0}, \chi\right)}{\nu} \geqslant \mu(V)-\varepsilon
$$

и теорема доказана. 
3. Случай конечного разбиения. Пусть теперь $\xi$ - конечное измеримое разбиение пространства $X$ с $q$ элементами.

Пусть $\gamma_{1}, \ldots, \gamma_{N}, 0 \leqslant \gamma_{i}<q, 1 \leqslant i \leqslant N,-$ некоторая система целых чисел, $0 \leqslant a<q$, $T_{N, a}-$ количество чисел, равных $a$, в этой системе. В работе [3] было доказано следующее утверждение.

УТВЕРЖДЕНИЕ 1. Существует некоторое положительное $\varepsilon_{1}=\varepsilon_{1}(\delta), \varepsilon_{1}(\delta) \rightarrow 0$, $\delta \rightarrow 0$, такое, что количество систем $c\left|T_{N, a}-N / q\right| \geqslant \delta N$ eсть $O\left(q^{N\left(1-\varepsilon_{1}\right)}\right)$.

Пусть монотонно возрастающая функция $\varphi$ представляется в виде $\varphi(t)=t \psi(t)$, где $\psi(t)$ - невозрастающая функция, которая удовлетворяет следующему условию: для произвольных $\delta>0, n \in \mathbb{N}$ и для всех характеристических функций $\chi$ элементов разбиения $\xi_{-n}^{T}$ вьполнено

$$
\lim _{l \rightarrow \infty} \max \left\{\mu\left(A_{l}\left(T^{n}, \chi, \delta\right)\right), \frac{1}{q^{l n \varepsilon_{1}(\delta)}}\right\} \psi\left(\frac{1}{q^{l n}}\right)=0 .
$$

ЗАмЕЧАНИЕ. Из условия, что для любых $\delta>0, n \in \mathbb{N}$ имеет место

$$
\lim _{l \rightarrow \infty} \frac{\psi\left(1 / q^{l n}\right)}{q^{l n \varepsilon_{1}(\delta)}}=0
$$

вытекает, что для $\varphi$ выполнено (2).

ТЕорема 2. Пусть точка $x_{0} \in X$ и ч удовлетворяет (10). Если для любого $n$ и характеристической функиии $\chi$ произвольного әлемента $I \in \xi_{-n}^{T}$ выполнено

$$
\limsup _{\nu \rightarrow \infty} \frac{S_{\nu}\left(x_{0}, \chi\right)}{\nu} \leqslant \mu(I) \psi(\mu(I)),
$$

то для характеристической функиии $\chi$ произвольного множества $E \in \Gamma$ выполнено

$$
\lim _{\nu \rightarrow \infty} \frac{S_{\nu}\left(x_{0}, \chi\right)}{\nu}=\mu(E) .
$$

ЗАмечаниЕ. Так как для динамической системы с $X=[0,1]$ и $T x=\{q x\}$

$$
\mu\left(A_{l}\left(T^{n}, \chi, \delta\right)\right)=O\left(\frac{1}{q^{\ln \varepsilon_{1}(\delta)}}\right)
$$

то из теоремы 2 следует теорема Б Пятецкого.

ДокАЗАТЕЛЬСтво. Возьмем любое $\delta>0$ и характеристическую функцию $\chi$ элемента $K$ разбиения $\xi$. Заметим, что множество $A_{l}(\chi, \delta)$ разбивается на объединение некоторых элементов разбиения $\xi_{-l}^{T}$. Проводим выкладку, аналогичную выкладке (5) из теоремы 1 . Для оценки слагаемого $R_{\nu} / \nu$ в правой части (5) нам нужен следующий аналог оценки (6):

$$
\limsup _{\nu \rightarrow \infty} \frac{R_{\nu}}{\nu} \leqslant \sum_{\lambda}^{*} \mu(\lambda) \psi(\mu(\lambda))
$$


где знаком $\sum_{\lambda}^{*}$ обозначено суммирование по всем элементам $\lambda$ разбиения $\xi_{-l}^{T}$, на которые разбивается $A_{l}(\chi, \delta)$.

Разобьем (13) на сумму $\sum_{1}$ по $\xi_{-l}^{T}(x)$ с мерами $<1 / q^{l}$ и на сумму $\sum_{2}$ по $\xi_{-l}^{T}(x)$ с мерами $\geqslant 1 / q^{l}$. По утверждению 1 количество слагаемьх в $(13)$ есть $O\left(q^{l\left(1-\varepsilon_{1}\right)}\right)$; следовательно, $\sum_{1} \ll \varphi\left(1 / q^{l}\right) q^{l\left(1-\varepsilon_{1}\right)}=\psi\left(1 / q^{l}\right) / q^{l \varepsilon_{1}(\delta)}$ (используем монотонность функции $\varphi$ ). Из того, что $\psi$ не возрастает, получаем $\sum_{2} \leqslant \mu\left(A_{l}(\chi, \delta)\right) \psi\left(1 / q^{l}\right)$. По условию (10) теоремы оба этих выражения $<\delta$ при достаточно большом $l$. Значит,

$$
\limsup _{\nu \rightarrow \infty}\left|\frac{S_{\nu}\left(x_{0}, \chi\right)}{\nu}-\mu(K)\right|=0
$$

т.e.

$$
\lim _{\nu \rightarrow \infty}\left|\frac{S_{\nu}\left(x_{0}, \chi\right)}{\nu}-\mu(K)\right|=0
$$

Пусть теперь $\chi$ - характеристическая функщия $E_{1} \cap T^{-1} E_{2} \cap \cdots \cap T^{-k+1} E_{k}, E_{i} \in \xi$, элемента $\xi_{-k}^{T}$ для некоторого $k$. Рассмотрим преобразование $T^{k}$ и будем считать разбиение $\eta=\xi_{-k}^{T}$ с $q^{k}$ элементами начальным для этой новой динамической системы. Условие (10) для этой динамической системы выполнено. Также вьполнено (7). Следовательно, применяя теорему $1^{\prime}$, получаем (12) для характеристической функции $\chi$ произвольного множества $E$ из Г. Теорема доказана.

4. О больших уклонениях. Для оценки мер множеств $A_{l}(\chi, \delta)$ бывают полезны центральная предельная теорема и оценки больших уклонений.

Пусть $\alpha=\left\{\alpha_{n}\right\}_{n=1,2, \ldots}$ - убывающая к нулю последовательность положительных чисел.

ОПредЕЛЕНИЕ. Динамическая система $(X, \Phi, \mu, T)$ с начальньм разбиением $\xi$ обладает свойством $\alpha$-перемешивания, если

$$
\sup _{M_{1} \in \xi_{-k}^{T}, M_{2} \in T^{-(n+k)}\left(\xi_{-\infty}^{T}\right)}\left\{\left|\mu\left(M_{1} \cap M_{2}\right)-\mu\left(M_{1}\right) \mu\left(M_{2}\right)\right|\right\} \leqslant \alpha(n) \mu\left(M_{1}\right)=\alpha_{n} \mu\left(M_{1}\right) .
$$

Пусть рассматриваемая динамическая система обладает свойством $\alpha$-перемешивания. Рассмотрим функцию $f(x)=\chi(x)-\int \chi d \mu$, где $\chi(x)$ - характеристическая функция произвольного элемента $\xi_{-n}^{T}$. Для таких систем выполнена

ШЕНТРАЛЬНАЯ ПРЕДЕЛЬНАЯ ТЕОРЕМА [11, гЛ. 4, §20]. Пусть $\sum \alpha_{n}^{1 / 2}<\infty u \sigma^{2}=$ $\mathrm{E} f^{2}+2 \sum_{k=1}^{\infty} \mathrm{E}\left(f \cdot f\left(T^{k}\right)\right)>0$. Тогда случайные величины $S_{n}(f, T) /(\sigma \sqrt{n})$ сходятся по распределению $к$ нормальной случайной величине $N\left(0, \sigma^{2}\right)$. (Символом Е обозначено математическое ожидание.)

Нам понадобятся теоремы больших уклонений из [13] для вероятностей $\mathrm{P}\left(x: \mid S_{l}(f\right.$, $\delta) / l \mid>\delta)$. Теоремы о больших уклонениях при наличии перемешивания устанавливают оценки следующего вида:

$$
\mu\left(A_{l}(\chi, \delta)\right)=\mathrm{P}\left(x:\left|\frac{S_{l}(f, \delta)}{l}\right|>\delta\right) \ll e^{-\rho(l)}
$$


с некоторой функцией $\rho(l)=\rho_{\delta}(l) \rightarrow \infty, l \rightarrow \infty$. Формулировки этих результатов в общем виде достаточно громоздки, поэтому в следующих двух пунктах мы сформулируем лишь необходимые нам следствия.

Отметим, что использованное нами утверждение 1 из п. 3 (принадлежащее Пятецкому-Шапиро) как раз является простейшим примером теоремы о больших уклонениях (см. [10, гл. $1, \S 6 ;$ гл. $4, \S 5])$.

5. Теорема Пятецкого для марковских цепей. Пусть матрица

$$
P=\left\{p_{i j}\right\}_{i, j=0, \ldots, n-1}
$$

стохастическая, т.е. такая, что $p_{i j} \geqslant 0$ и $\sum_{j} p_{i j}=1$. Матрица $P$ назьвается транзитивной, если для некоторого $m$ все элементы матрищы $P^{m}$ положительны. Тогда [1], [10] сушествует вектор $\pi=\left(\pi_{1}, \ldots, \pi_{n}\right)$ с положительными компонентами такой, что $P^{*} \pi=\pi\left(P^{*}-\right.$ транспонированная матрица).

Рассмотрим пространство бесконечных вправо последовательностей $\Omega$, составленных из знаков $0, \ldots, n-1$. Меру $\mu$ элементарного цилиндра, т.е. множества, у которого фиксировано какое-то количество первых знаков, положим равной $\pi_{\delta_{1}}$, если фиксирован первый знак $\delta_{1}$, и равной $\pi_{\delta_{1}} p_{\delta_{1} \delta_{2}} \cdots p_{\delta_{s-1} \delta_{s}}$, если фиксированы знаки $\delta_{1}, \ldots, \delta_{s}$. Мера пустого множества равна 0 , всего пространства - 1 . Эту меру можно продолжить на минимальную $\sigma$-алгебру, содержащую алгебру цилиндрических множеств. Легко проверить, что полученная таким образом мера $\mu$, будет инвариантна относительно сдвига влево T. Это преобразование эргодично (см. [1], [12]). Конечная транзитивная цепь Маркова обладает свойством $\alpha$-перемешивания с $\alpha_{n}=a e^{-b n}, a, b>0$ (см. [11, гл. 4, $\S 20])$. Более того, для мер множеств $A_{l}\left(T^{k}, \chi, \delta\right)$, где $\chi(x)$ - характеристическая функция элементарного цилиндра с $k$ фиксироваными знаками, вьполнена оценка (см. [13, лемма 2.4 и теорема 4.26$]$ )

$$
\mu\left(A_{l}(\chi, \delta)\right) \ll e^{-c l}
$$

с некоторым $c=c(\delta)>0$.

Теорема 3. Пусть $x_{0} \in \Omega$. Пусть также для всякого полохительного $\eta$ выполнено $\psi(t)=O\left(t^{-\eta}\right), t \rightarrow 0$. Если для любого элементарного иилиндра I выполнено

$$
\limsup _{\nu \rightarrow \infty} \frac{S_{\nu}\left(x_{0}, I\right)}{\nu} \leqslant \mu(I) \psi(\mu(I)),
$$

то для произвольного множества $V$ из Г выполняется

$$
\lim _{\nu \rightarrow \infty} \frac{S_{\nu}\left(x_{0}, V\right)}{\nu}=\mu(V)
$$

ДокАЗАтЕльство. Рассмотрим разбиение пространства $\Omega$ на элементарные цилиндры с первьм фиксированным знаком. Возьмем любой элементарньй цилиндр $I_{k}, \mathrm{y}$ которого $k$ первых знаков фиксировано. Обозначим через $\chi$ его характеристическую функцию, и пусть $f(x)=\chi(x)-\int \chi d \mu$. Из оценки (15) имеем

$$
\lim _{l \rightarrow \infty} \max \left\{\mu\left(A_{l}\left(T^{k}, \chi, \delta\right)\right), \frac{1}{n^{l k \varepsilon_{1}(\delta)}}\right\} \psi\left(\frac{1}{n^{l k}}\right)=0 .
$$

Применяя теорему 2 , получаем теорему 3 . 
6. Примеры со счетным разбиением. В следующих двух примерах динамических систем со счетным начальньм разбиением $\xi$ можно явно указать функцию $\varphi$.

а) Первьй пример относится к теории цепньх дробей.

Цепной дробъю, соответствующей числу $\alpha$, назьвается выражение

$$
\alpha=a_{0}+\frac{1}{a_{1}+\frac{1}{a_{2}+\cdots}}=\left[a_{0} ; a_{1}, a_{2}, \ldots\right] .
$$

Число $a_{n}$ назьвается $n$-м неполным частным числа $\alpha$, а рациональное число $p_{k} / q_{k}=$ $\left[a_{0} ; a_{1}, a_{2}, \ldots, a_{k}\right]-$ подходящей дробъю порядка $k$. Если $\alpha \in[0,1)$, то будем писать $\alpha=\left[a_{1}, a_{2}, \ldots\right]$. Интервалом $k$-го ранга $I_{k}=I_{k}\left(a_{1}, \ldots, a_{k}\right)$ называется множество всех чисел $\alpha$, у которых цепные дроби начинаются с $a_{1}, \ldots, a_{k}$. Обозначим через $S_{\nu}\left(\alpha, I_{k}\right)$ число повторений комбинации $\left(a_{1}, \ldots, a_{k}\right)$ в разложении $\alpha$ до $\nu$-го места.

Как известно, преобразование Гаусса $T x=\{1 / x\}, x \neq 0, T 0=0$, является левьм сдвигом при разложении в цепную дробь и сохраняет меру

$$
\mu(A)=\frac{1}{\ln 2} \int_{A} \frac{1}{1+x} d x
$$

которая эквивалентна мере Лебега $d x$. Соответствующая динамическая система обладает свойством $\alpha$-перемешивания (теорема Леви, см. [11, гл. 4, §20], а также [9, примечание, с. 83]) с $\alpha_{n}=K e^{-\gamma n}, K, \gamma>0$. В рассматриваемом случае теорема больших уклонений для слабо зависимых случайных величин (см. [13, лемма 2.4 и теорема 4.23]) обеспечивает оценки для мер

$$
\mu\left(A_{l}(\chi, \delta)\right) \ll e^{-c \sqrt{l}}
$$

с некоторым $c=c(\delta)>0$.

Теорема 4. Пусть $x_{0} \in[0,1)$. Пусть также для всякого полохительного $\eta$ выполнено $\psi(t)=O\left(e^{\eta \sqrt{\log 1 / t}}\right), t \rightarrow 0$. Если для любого интервала $k$-го ранга $I_{k}$ имеет место неравенство

$$
\limsup _{\nu \rightarrow \infty} \frac{S_{\nu}\left(x_{0}, I_{k}\right)}{\nu} \leqslant \mu\left(I_{k}\right) \psi\left(\mu\left(I_{k}\right)\right)
$$

то для любого интервала $I=(a, b)$ выполнено

$$
\lim _{\nu \rightarrow \infty} \frac{S_{\nu}\left(x_{0}, I\right)}{\nu}=\mu(I)
$$

ДоКАЗАТЕЛЬСТво. Возьмем любое $\delta>0$, и пусть $I_{k}-$ произвольньй интервал $k$-го ранга. Обозначим через $E_{l}(g)(g \geqslant 1)$ множество чисел отрезка $[0,1]$, для которых $a_{1} a_{2} \cdots a_{l} \geqslant g$. Это множество представляет собой систему интервалов ранга $l$. Длина $\left|I_{l}\right|$ какого-либо из этих интервалов равна $1 /\left(q_{l}\left(q_{l}+q_{l-1}\right)\right)$ и $1 /\left(2 q_{l}^{2}\right) \leqslant\left|I_{l}\right| \leqslant 1 / q_{l}^{2}$. Далее, по индукции получаем, что $a_{1} a_{2} \cdots a_{l} \leqslant q_{l} \leqslant 2^{l} a_{1} a_{2} \cdots a_{l}$.

Оценим хаусдорфову меру $E_{l}(g)$ относительно функции $\varphi$, т.е. сумму

$$
H_{\varphi}\left(E_{l}(g)\right)=\sum_{a_{1} a_{2} \cdots a_{l} \geqslant g}\left|I_{l}\right| \psi\left(\left|I_{l}\right|\right) .
$$


Обозначая $t^{-\eta}$ через $\rho(t)$, имеем

$$
H_{\varphi}\left(E_{l}(g)\right) \leqslant \sum_{a_{1} a_{2} \cdots a_{l} \geqslant g} \frac{\rho\left(\left(2^{l+1} a_{1} a_{2} \cdots a_{l}\right)^{2}\right)}{\left(a_{1} a_{2} \cdots a_{l}\right)^{2}}=\rho\left(4^{l+1}\right) \sum_{a_{1} a_{2} \cdots a_{l} \geqslant g} \prod_{i=1}^{l} \frac{\rho\left(a_{i}^{2}\right)}{a_{i}^{2}} .
$$

По аналогии с рассуждениями из [9] получим оценку для (17). Заметим, что

$$
\prod_{i=1}^{l} \frac{\rho\left(a_{i}^{2}\right)}{a_{i}^{2}}=\prod_{i=1}^{l}\left(1+\frac{1}{a_{i}}\right) \frac{\rho\left(a_{i}^{2}\right)}{a_{i}\left(a_{i}+1\right)} \leqslant 2^{l} \prod_{i=1}^{l} \int_{a_{i}}^{a_{i}+1} \frac{\rho\left(x_{i}^{2}\right)}{x_{i}^{2}} d x_{i} .
$$

Следовательно,

$$
H_{\varphi}\left(E_{l}(g)\right) \leqslant 2^{l} \rho\left(4^{l+1}\right) J_{l}(g)=2^{l} \rho\left(4^{l+1}\right) \int \cdots \int \frac{\rho\left(x_{1}^{2} \cdots x_{l}^{2}\right) d x_{1} \cdots d x_{l}}{x_{1}^{2} \cdots x_{l}^{2}},
$$

где интегрирование распростаняется на область $x_{i} \geqslant 1, x_{1} x_{2} \cdots x_{l} \geqslant g$. По индукции доказьвается, что

$$
J_{n}(g)=\frac{\rho\left(g^{2}\right)}{g}\left(\sum_{k=0}^{n-2} C^{n-k-1} \frac{\log ^{k} g}{k !}+C \frac{\log ^{n-1} g}{(n-1) !}\right)
$$

где $C=1 /(1-2 \eta)$. Дальнейший подсчет показывает, что, полагая $g=e^{A l}$, где $A$ достаточно большое, имеем

$$
H_{\varphi}\left(E_{l}\left(e^{A l}\right)\right) \ll e^{-B l}, \quad B>0 .
$$

Далее,

$$
\limsup _{\nu \rightarrow \infty} \frac{R_{\nu}}{\nu} \leqslant \sum_{I}^{* *} \mu(I) \psi(\mu(I))
$$

где, в отличие от теоремы 2 (ср. с формулой (13)), суммирование $\sum_{I}^{* *}$ распространяетсяна счетное количество интервалов $k$-го ранга $I$. Разобьем (19) на сумму по $I_{l} \subseteq E_{l}(g)$ и на сумму по $I_{l} \subseteq A_{l}\left(I_{k}, \delta\right) \backslash E_{l}(g)$. Так как для $I_{l}$, не принадлежащих $E_{l}(g)$, имеет место неравенство $\left|I_{l}\right| \geqslant \frac{1}{2}\left(2^{l} g\right)^{2}=1 / e^{K l}$, где $K>0$ - некоторая новая константа, используя (18), мы получаем, что

$$
\limsup _{\nu \rightarrow \infty} \frac{R_{\nu}}{\nu} \ll e^{-B l}+\psi\left(\frac{1}{e^{K l}}\right) \mu\left(A_{l}\left(I_{k}, \delta\right)\right) .
$$

Из оценки (16) следует, что при достаточно малом $\eta$ правая часть (20) стремится к 0 при $\nu \rightarrow \infty$. Значит, для характеристической функции $\chi$ интервала $I_{k}$ вьполнено

$$
\limsup _{\nu \rightarrow \infty}\left|\frac{S_{\nu}\left(x_{0}, \chi\right)}{\nu}-\mu\left(I_{k}\right)\right|=0,
$$

т.е.

$$
\lim _{\nu \rightarrow \infty}\left|\frac{S_{\nu}\left(x_{0}, \chi\right)}{\nu}-\mu\left(I_{k}\right)\right|=0
$$


Возьмем теперь произвольный интервал $(a, b)$. Так как длины интервалов $I_{n}$ убывают, то $(a, b)$ может быть аппроксимирован с любой точностью такими интервалами. Теорема 4 доказана.

б) Второй пример является обобщением теоремы Пятецкого-Шапиро на случай системы с бесконечным алфавитом.

Пусть $p_{a}, a=1,2,3, \ldots,-$ положительные числа, $\sum_{a=1}^{\infty} p_{a}=1$. Рассматривается пространство $\Omega$ бесконечных вправо последовательностей

$$
\xi=\left(\xi_{1}, \ldots, \xi_{\nu}, \ldots\right)
$$

с мерой $\mu$, определенной на элементарных цилиндрических множествах

$$
\Delta\left(a_{1}, \ldots, a_{n}\right)=\left\{\xi: \xi_{j}=a_{j}, j=1, \ldots, n\right\}
$$

по правилу $\mu \Delta\left(a_{1}, \ldots, a_{n}\right)=p\left(a_{1}, \ldots, a_{n}\right)=p_{a_{1}} \cdots p_{a_{n}}$ и продолженной на всю $\sigma$-алгебру, порожденную цилиндрическими множествами. Для набора $a=\left(a_{1}, \ldots, a_{n}\right)$ будем использовать обозначение $p(a)=p\left(a_{1}, \ldots, a_{n}\right)$. Преобразовние сдвига влево мы будем снова обозначать $T$ (хорошо известно, что оно эргодично).

ТеОРема 5. Пусть при некотором положительном $\eta_{0}$ ряд

$$
\sum_{a=1}^{\infty} p_{a}^{1-\eta_{0}}
$$

сходится. Пусть $\varphi(t)$ - произвольная положительнозначная функиия, стремящаяся $к 0$ при $t \rightarrow 0$ и такая, что для всякого положительного $\eta$ выполнено $\varphi(t)=O\left(t^{1-\eta}\right), t \rightarrow 0$. Если для любого әлементарного иилиндра I выполнено

$$
\limsup _{\nu \rightarrow \infty} \frac{S_{\nu}\left(x_{0}, \chi_{I}\right)}{\nu} \leqslant \varphi(\mu(I))
$$

то для характеристической функции х произвольного множества $E \in \Gamma$

$$
\lim _{\nu \rightarrow \infty} \frac{S_{\nu}\left(x_{0}, \chi\right)}{\nu}=\mu(E) .
$$

ДокаЗАТЕЛЬСтво. Определим

$$
\sigma_{n, \delta}(p, q ; x, y)=\sum_{k: k / n \notin[p-\delta, p+\delta]} C_{n}^{k} p^{(1-x) k} q^{(1-y)(n-k)}
$$

и для набора $a=\left(a_{1}, \ldots, a_{t}\right)$ величину

$$
\sigma_{n, \delta}(a, \eta)=\sum_{k: k / n \notin[p(a)-\delta, p(a)+\delta]} C_{n}^{k}\left(p(a)^{(1-\eta)}\right)^{k} \cdot\left(\sum_{b=\left(b_{1}, \ldots, b_{t}\right) \neq a} p(b)^{1-\eta}\right)^{n-k} .
$$


Лемма. Для любых вещественных чисел $p, q>0, p+q=1$, существует постоянная $\delta_{0}(p, q)$ такая, что для кажсдого положительного $\delta<\delta_{0}(p, q)$ найдутся величины

$$
w_{\delta}(p, q), \varepsilon_{\delta}(p, q), n_{\delta}(p, q)
$$

такие, что при всех $n>n_{\delta}(p, q)$ выполнено

$$
\sup _{x, y \in\left[0, w_{\delta}(p, q)\right]} \sigma_{n, \delta}(p, q ; x, y)<\exp \left(-\varepsilon_{\delta}(p, q) n\right) .
$$

Доказательство леммы полностью аналогично соотетствующему утверждению из [3]: надо заметить, что максимум функции $g(k)=C_{n}^{k} p_{1}^{k} q_{1}^{n-k}$ достигается при

$$
\frac{k}{n} \sim \frac{p_{1}}{p_{1}+q_{1}}
$$

(и при переходе через этот максимум возрастание сменяется убыванием) и что

$$
\sigma_{n, \delta}(p, q ; x, y) \leqslant\left(\kappa_{\delta}^{(+)}(p, q, ; x, y,)\left(1+O\left(\frac{1}{n}\right)\right)\right)^{n}+\left(\kappa_{\delta}^{(-)}(p, q, ; x, y)\left(1+O\left(\frac{1}{n}\right)\right)\right)^{n}
$$

где

$$
\kappa_{\delta}^{( \pm)}(p, q, ; x, y)=\frac{p^{(1-x)(p \pm \delta)} q^{(1-y)(q \mp \delta)}}{(p \pm \delta)^{p \pm \delta}(q \mp \delta)^{q \mp \delta}}
$$

- непрерьвная в нуле функция переменных $x, y$ и

$$
\lim _{x, y \rightarrow 0} \kappa_{\delta}^{( \pm)}(p, q, ; x, y,)=1-\frac{\delta^{2}}{2}\left(\frac{1}{p}+\frac{1}{q}\right)+O\left(\delta^{3}\right), \quad \delta \rightarrow 0 .
$$

СЛЕДСТВИЕ ЛЕммЫ. Пусть при некотором положительном $\eta_{0}$ ряд

$$
\sum_{a=1}^{\infty} p_{a}^{1-\eta_{0}}
$$

сходится. Тогда для любого набора $a=\left(a_{1}, \ldots, a_{t}\right)$ найдется положительное $\delta(a)$ такое, что для каждого положительного $\delta<\delta(a)$ найдутся положительные

$$
\eta(\delta, a), \varepsilon(\delta, a), n(\delta, a)
$$

такие, что при всех $\eta \in[0, \eta(\delta, a)]$ и при всех $n>n_{\delta}(p, q)$ выполнено

$$
\sigma_{n, \delta}(a, \eta)<\exp (-\varepsilon(\delta, a) n)
$$

Для того чтобы получить сформулированное следствие, полагаем $p=p(a), q=$ $1-p(a)$ и используем, что функции $f(\eta)=p(a)^{1-\eta}$ и $g(\eta)=\sum_{b: b \neq a}(p(b))^{1-\eta}$ при $\eta$ близких к нулю являются непрерьвными; в частности, на некотором интервале $0 \leqslant \eta \leqslant$ $\eta(\delta, a)$ имеем $g(0)=1-p(a) \leqslant g(\eta) \leqslant(1-p(a))^{w_{\delta}(p, q)}$. Затем применяем лемму.

Если теперь рассмотреть характеристическую функцию $\chi_{a}$ элементарного цилиндра с фиксированными первыми знаками $a=\left(a_{1}, \ldots, a_{t}\right)$, то при достаточно большом $n$ будет иметь место $H_{\varphi}\left(A_{n}\left(\chi_{a}, \delta\right)\right) \ll \sigma_{n, \delta}(a, \eta)$. Теперь согласно $(22) H_{\varphi}\left(A_{n}\left(\chi_{a}, \delta\right)\right) \rightarrow 0$, $n \rightarrow \infty$, и теорема 5 вытекает из теоремы 1 .

Теорема 5 доказана. 


\section{7. Замечания.}

Замечание 1 . Теоремы $1,1^{\prime}$ носят общий характер.

ЗАмЕчАниЕ 2. Теоремы 3-5 имеют дело с конкретными динамическими системами. Легко видеть, что теоремы 3 и 5 неулучшаемы, т.е. ослабить условие на функцию $\psi$ в этих теоремах нельзя (см. [3]). В теореме 4 вместо условия $\varphi(t)=O\left(t e^{-\eta \sqrt{\log 1 / t}}\right)$, $t \rightarrow 0$, наверное, можно потребовать лишь условие $\varphi(t)=O\left(t^{1-\eta}\right), t \rightarrow 0$, но доказать это мы не можем.

ЗАмечаниЕ 3. Теоремы 3, 4 доказаны нами с использованием сложных теорем о больших уклонениях [13]. Теорема 5 тоже может быть выведена из теоремы о больших уклонениях для сумм независимых случайных величин (см. [10, гл. 4, §5]), однако по причине ее простоты мы привели элементарное доказательство.

ЗАмЕчАниЕ 4. Не вызывает сомнения, что подобного рода усиленньй критерий Пятецкого-Шапиро справедлив и в задаче о распределении дробных долей матричной показательной функции, рассматривавшейся в [5].

\section{СПИСОК ЦИТИРОВАННОЙ ЛИТЕРАТУРЫ}

[1] Каток А. Б., Хасселблат Б. Введение в современную теорию динамических систем. М.: Изд-во "Факториал", 1999.

[2] Пятецкий-Шапиро И.И.О законах распределения дробных долей показательнй функции // Изв. АН СССР. Сер. матем. 1951. Т. 15. С. 47-52.

[3] Пятецкий-Шапиро И.И. О расспределении дробных долей показательной функции // Ученые записки МГПИ. 1957. Т. 58. № 2. С. 317-322.

[4] Постников А. Г. Арифметическое моделирование случайных процессов. Тр. МИАН. Т. 57. М.: Наука, 1960.

[5] Постников А. Г. Эргодические вопросы теории сравнений и теории диофантовых приближений. Тр. МИАН. Т. 82. М.: Наука, 1966.

[6] Schmidt W. M. Badly approximable numbers and certain games // Trans. Amer. Math. Soc. 1966. V. 123. P. 178-199.

[7] von Neumann J. Physical applications of the ergodic hypothesis // Proc. Nat. Acad. Sci. 1932. V. 18. P. 263-266.

[8] Качуровский А. Г. Скорости сходимости в эргодических теоремах // УМН. 1996. Т. 51. № 4. C. $73-124$.

[9] Хинчин А. Я. Цепные дроби. М.: Наука, 1978.

[10] Ширяев А. Н. Вероятность. М.: Наука, 1989.

[11] Биллингсли П. Сходимость вероятностных мер. М.: Наука, 1977.

[12] Романовский В. И. Дискретные цепи Маркова. М.: Гостехиздат, 1949.

[13] Саулис Л., Статулявичус В. Предельные теоремы о больших уклонениях. Вильнюс: Мокслас, 1989.

Московский государственный университет им. М.В. Ломоносова

E-mail: moshche@mech.math.msu.su, ishkredov@rambler.ru 